\title{
NuSTAR hard x-ray optics
}

Jason E. Koglin, Finn E. Christensen, William W. Craig, Todd R. Decker, Charles J. Hailey, et al.

Jason E. Koglin, Finn E. Christensen, William W. Craig, Todd R. Decker, Charles J. Hailey, Fiona A. Harrison, Colin Hawthorn, Carsten P. Jensen, Kristin K. Madsen, Marcela Stern, Gordon Tajiri, Michael D. Taylor, "NuSTAR hard x-ray optics," Proc. SPIE 5900, Optics for EUV, X-Ray, and Gamma-Ray Astronomy II, 59000X (1 September 2005); doi: 10.1117/12.618601 


\title{
NuSTAR Hard X-ray Optics
}

\author{
Jason E. Koglin ${ }^{\mathrm{a}^{*}}$, Finn E. Christensen ${ }^{\mathrm{c}}$, William W. Craig ${ }^{\mathrm{d}}$, Todd R. Decker ${ }^{\mathrm{d}}$, \\ Charles J. Hailey ${ }^{\mathrm{a}}$, Fiona A. Harrison ${ }^{\mathrm{b}}$, Colin Hawthorn ${ }^{\mathrm{a}}$, Carsten P. Jensen ${ }^{\mathrm{c}}$, \\ Kristin K. Madsen ${ }^{\mathrm{c}}$, Marcela Stern ${ }^{\mathrm{a}}$, Gordon Tajiri ${ }^{\mathrm{a}}$, Michael D. Taylor ${ }^{\mathrm{e}}$ \\ ${ }^{\mathrm{a} C}$ Columbia Astrophysics Laboratory, New York, NY \\ ${ }^{b}$ California Institute of Technology, Pasadena, CA \\ ${ }^{c}$ Danish National Space Center, Copenhagen, Denmark \\ ${ }^{\mathrm{d}}$ Lawrence Livermore National Laboratory, Livermore, CA \\ ${ }^{\mathrm{e}}$ Jet Propulsion Laboratory, Pasadena, CA
}

\begin{abstract}
The Nuclear Spectroscopic Telescope Array (NuSTAR) is a small explorer (SMEX) mission currently under an extended Phase A study by NASA. NuSTAR will be the first satellite mission to employ focusing optics in the hard X-ray band (8$80 \mathrm{keV}$ ). Its design eliminates high detector backgrounds, allows true imaging, and permits the use of compact high performance detectors. The result: a combination of clarity, sensitivity, and spectral resolution surpassing the largest observatories that have operated in this band by orders of magnitude. We present an overview of the NuSTAR optics design and production process. We also describe the progress of several components of our independent optics development program that are beginning to reach maturity and could possibly be incorporated into the NuSTAR production scheme. We then present environmental test results that are being conducted in preparation of full space qualification of the NuSTAR optics.
\end{abstract}

Keywords: Hard X-ray telescope, X-ray optics, segmented glass, thermally-slumped, HEFT, NuSTAR

\section{EXPLORING THE HARD X-RAY UNIVERSE}

The Nuclear Spectroscopic Telescope Array (NuSTAR) is a pathfinder mission that will open the hard X-ray frontier. Addressing key parts of the NASA strategic plan, NuSTAR will answer fundamental questions about our Universe:

- How are black holes distributed through the cosmos, and how do they influence the formation of galaxies like our own?

- How were the elements that compose our bodies and the Earth forged in the explosions of massive stars?

- What powers the most extreme active galaxies?

A quarter century ago, the Einstein mission introduced high-resolution focusing optics to X-ray astronomy. By eliminating high detector backgrounds, allowing true imaging, and permitting the use of compact highperformance detectors, Einstein leapt a factor of a hundred in sensitivity, completely changing our view of the X-ray sky. Now NuSTAR is poised to make the same jump in the high-energy X-ray band, offering a combination of clarity, sensitivity, and spectral resolution surpassing the largest observatories in this band by orders of magnitude (c.f., continuum sensitivity plot in Figure 1). NuSTAR is designed to address three specific high priority questions, but history shows that terra incognita often

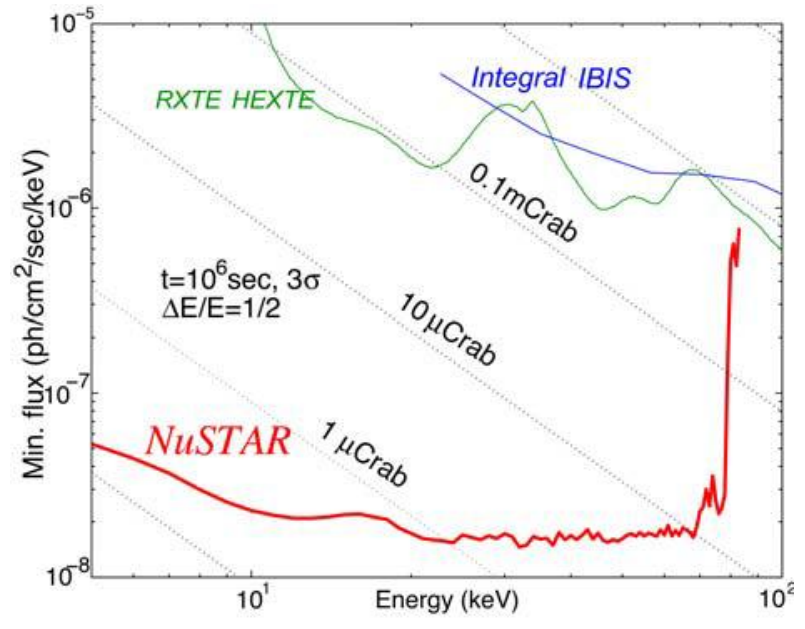

Figure 1: NuSTAR continuum sensitivity plotted versus energy together with other hard X-ray instruments.

\footnotetext{
${ }^{*}$ Correspondence: koglin@astro.columbia.edu, http://www.astro.columbia.edu/ koglin
} 
hides spectacular unexpected discoveries. The Sloan Digital Sky Survey has revolutionized optical astronomy by looking ten times deeper than previous surveys, with a vision three times as sharp. In contrast, NuSTAR will surpass previous hard X-ray surveys by more than a factor of a hundred in both depth and resolution. In its waveband, NuSTAR's advances are comparable to Spitzer over ISO in the infrared, or GLAST over CGRO in gamma-rays.

In pursuit of these science objectives, NuSTAR will:

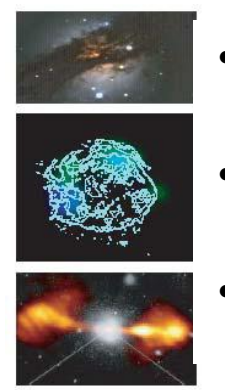

- Conduct a census for black holes on all scales, achieved through deep, wide-field surveys of extragalactic fields and the Galactic center,

- Map radioactive material in young supernova remnants to study the birth of the elements and to understand how stars explode,

- Explore relativistic jets of particles from the most extreme active galaxies to understand what powers giant cosmic accelerators.

NuSTAR will also study cosmic ray origins and the extreme physics around collapsed stars and will respond to targets of opportunity including supernovae and gamma-ray bursts.

NuSTAR builds on the High Energy Focusing Telescope (HEFT) balloon program, using a simple design with extensive heritage. The focusing optics that we have developed at Columbia, together with pixelized solid state detectors developed by CalTech, will make NuSTAR 1000 times more sensitive than previous experiments. NASA selected NuSTAR in November 2003 as one of five finalists for two small explorer satellite missions. NASA recently selected NuSTAR for continued study for the second satellite mission scheduled for early 2009. A formal decision on proceeding to flight development for NuSTAR will be made by early 2006 .

\section{NUSTAR SATELLITE OVERVIEW}

NUSTAR consists of a single instrument that achieves its science objectives with a combination of surveys and pointed observations. The mission objective is to return the required data to meet the science goals. The robust mission design is based on a 3-axis stabilized spacecraft and simple operations concept. A Pegasus launch to equatorial orbit provides a stable, low-background environment.

\subsection{Instrument Overview}

The NuSTAR instrument consists of an array of three coaligned hard X-ray telescopes. The grazing incidence mirrors focus onto three shielded solid-state pixel detectors, separated by a mast that extends the focal length to 10 meters after launch. A laser metrology system monitors the mast alignment. The optics utilize thin glass shells coated with depth-graded multi-layers to extend the bandpass and field of view (FOV) over that achievable with standard metal surfaces. Cadmium Zinc Telluride (CdZnTe) detectors provide excellent spectral resolution and high quantum efficiency without requiring cryogenic operation. The detectors and their associated electronics are housed in $\mathrm{Pb}$ /plastic scintillator shielding. An illustration of the partially deployed instrument is shown in Figure 2 and an overview of the instrument configuration is given in Table 1.

The NUSTAR focal plane (including the CdZnTe detectors, shielding and associated electronics) is directly based on the HEFT focal plane, ${ }^{1}$ and in most cases no modifications are required, giving high

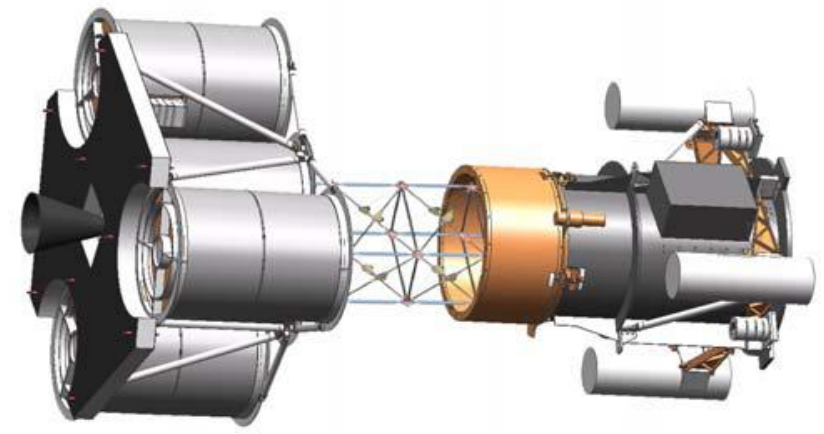

Figure 2: NuSTAR instument (partially deployed).

Table 1: NuSTAR instrument configuration.

\begin{tabular}{|l|l|}
\hline Parameter & Value \\
\hline \# telesc. modules & 3 \\
\hline Eff. focal length & $10.03 \mathrm{~m}$ \\
\hline Optics & Grazing incidence. Conical approx. \\
\hline Reflecting surface & W/SiC\&Pt/C graded multilayers \\
\hline Sensors & CdZnTe pixel hybrid \\
\hline Shielding & Pb/plastic scintillator \\
\hline
\end{tabular}


confidence in the performance characteristics. The optics design and proposed production process for NuSTAR is very similar to that used to successfully build three HEFT telescopes. The HEFT optics design and production process has been described in detail elsewhere. ${ }^{2,3,4}$ We have also previously given an overview of the NuSTAR optics design and outlined how our experience in manufacturing complete HEFT telescopes, as well as our experience developing higher performance prototype optics, will lead to the successful production of telescopes that meet the NuSTAR design goals. ${ }^{5}$

\subsection{Optics Design}

The three optical modules on NUSTAR collect the incoming hard X-rays and focus them on to the detector. The optical design is a conical approximation to a Wolter-I, providing high performance across the 12' maximum field of view. The optics (c.f., Figure 3), are built of thermally formed glass substrates, precisely held in position by epoxy and graphite spacers.

Each of the 130 shells in an optic is made up of 32 substrates ( 8 in azimuth and 4 along the optic axis). Three flight optics modules and one spare require 16,640 substrates, manufactured from $200 \mu \mathrm{m}$ thick glass. Details of the instrument design are provided in Table 2. Depth-graded multilayers are applied to the optical surfaces in a production coating facility at the Danish National Space Center (DNSC) $)^{\dagger}$ that uses planar magnetron sputtering. The specific multilayer design depends on graze angle, which scales with the optic radius - the inner 14 radius groups (about 2/3 of the geometric area) are coated with $\mathrm{Pt} / \mathrm{SiC}$, and the remaining groups are coated with $\mathrm{W} / \mathrm{SiC}$. Further details of multilayers proposed for NuSTAR are detailed elsewhere. ${ }^{6}$

The entire assembly is built on, and aligned to, a central mandrel. After construction, an external can, to protect the optics during subsequent handling, and a support spider are attached. Alignment pins are added during optics calibration and used to tie the optical modules to the optical bench during instrument integration. Each telescope has two end fittings that provide handling and attachment features and carry loads between the inner, intermediate, and outer mandrels. During fabrication, glass and graphite layers are built outward from the central mandrel and all ground handling and alignment activities use the inner mandrel as a support and reference point. Once the telescopes are mounted to the telescope bench, each is supported and aligned from attachment points on the outer mandrel. The end fittings provide a structural link back to the center mandrel and distribute loading across the telescope end faces.

NuSTAR will be the first satellite mission to employ focusing optics in the hard X-ray band, extending the reach of current soft X-ray instruments by nearly a decade in energy. The total on-axis effective area for NuSTAR is plotted as a function of energy in Figure 4 and compared to current focusing instruments. The focusing optics of NuSTAR deliver a dramatic improvement in sensitivity over the previous generation of coded aperture and collimated optics used for hard X-rays. The three-sigma continuum sensitivity $(\Delta \mathrm{E} / \mathrm{E}=0.5)$ achieved from a $10^{6}$ second observation with $N u S T A R$ is plotted versus energy in Figure 1 and compared with current hard X-ray observatories.

Table 2: Telescope parameters

\begin{tabular}{|l|l|}
\hline \multicolumn{2}{|c|}{ Mirror Parameters } \\
\hline Geometry & Segmented conical approx. \\
\hline Substrates & Thermally formed glass \\
\hline Shells/module & 130 \\
\hline Shell thickness & $0.2 \mathrm{~mm}$ \\
\hline Shell length & $400 \mathrm{~mm}$ \\
\hline Inner radius & $54.9 \mathrm{~mm}$ \\
\hline Outer radius & $168.6 \mathrm{~mm}$ \\
\hline Focal Length & $10.037 \mathrm{~m}$ \\
\hline \multicolumn{2}{|c|}{ Multilayer Parameters } \\
\hline Materials & W/SiC (outer shells) \\
& Pt/C (inner shells) \\
\hline Design & Depth graded \\
\hline Max. thickness & $1.6(\mathrm{~W} / \mathrm{SiC}) 0.6 \mu \mathrm{m}(\mathrm{Pt} / \mathrm{C})$ \\
\hline Max. layer pairs & $550(\mathrm{~W} / \mathrm{Si}) 150(\mathrm{Pt} / \mathrm{C})$ \\
\hline \multicolumn{2}{|c|}{ Focal Plane (FP) Module Parameters } \\
\hline CdZnTe Pixel pitch & $0.5 \mathrm{~mm}$ \\
\hline CZT Units/FP & 2 \\
\hline Unit dimensions & $2 \times 13 \times 25 \mathrm{~mm}$ \\
\hline Pb/plastic shield thick. & $2 \mathrm{~mm} / 1.5 \mathrm{~cm}$ \\
\hline
\end{tabular}

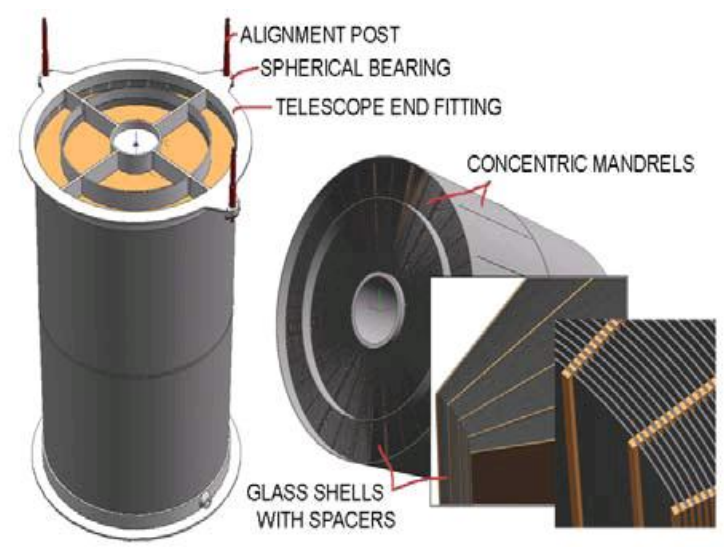

Figure 3: NuSTAR optics design.

\footnotetext{
${ }^{\dagger}$ Formerly the Danish Space Research Institute (DSRI)
} 


\subsection{Substrate Production}

Our approach to the mirror fabrication depends on keeping all aspects of production uncomplicated, relying primarily on off-the-shelf hardware. The process begins by slumping glass microsheets into standard quartz mandrels using commercial ovens. A laser scanning system is used to measure the performance of the thermally formed glass. The laser scanner data is used as a metric for substrate selection and as a feedback mechanism in tuning the oven settings. Only small fine tuning adjustments are required over weeks of mass production slumping with the same setup. The central portion of the $20 \mathrm{~cm}$ long slumped shells is cut out and a multilayer coating is applied to the inside reflecting surface before it is mounted. The substrate production process relies primarily on unskilled technicians for slumping and scanning.

The slumping process is not strictly deterministic and results in an ensemble of substrates with a broad distribution in performance. For HEFT it was not possible to scan every substrate, and sample metrology was performed only periodically for general quality assurance. Nearly every substrate was accepted for $H E F T$ - a majority of which with no knowledge of the actual freestanding performance. The central $72^{\circ}$ portion of the thousands of slumped substrates that were scanned for HEFT have ranged in performance from $\sim 15^{\prime}$ to over 4' with a mean of $\sim 1$ '. By accepting nearly every substrate we produced, we achieved a composite $\sim 1$ arcminute performance for HEFT.

Our strategy for NuSTAR requires every substrate to be scanned and relies on selecting a smaller portion $\left(45^{\circ}\right.$ octant geometry instead of the $72^{\circ}$ quintant geometry used for HEFT) of only the best pieces of glass (See reference 7 for details). We have demonstrated the NUSTAR goal of 40" by selecting the best half of a random selection of substrates (i.e., $50 \%$ glass yield). We have already increased our capacity for manufacturing substrates by putting eight more ovens online at Columbia's Nevis Laboratory (for a total of 20 ovens). We have nearly completed the design of a new scanning machine capable of fully characterizing a substrate in less than ten minutes (approximately two times faster than our current machine). The first of three new scanning machines planned for NuSTAR will be put online before the end of 2005 .

We are designing equipment that employs a hot wire method to cut the glass substrates. This method, which was developed at GSFC and has been successfully prototyped at Columbia, will replace the scribe and break method that was used for HEFT. While the scribe and break method, which was used to cut over 5000 substrates with $92 \%$ yield for $H E F T$, would be sufficient for NuSTAR, the wire cutting method produces a much cleaner cut and will further reduce the chance of fracture during subsequent handling and will improve overall quality assurance.

The HEFT program has produced more than 5000 flight mirror segments (over 12,500 total including prototypes and those produced in process development for NuSTAR). The NuSTAR substrate production is based on the procedures and lessons learned from $H E F T$, with upgrades in production facilities where required. Based on the experience of $H E F T$, a combined yield for glass selection, handling and cutting is expected to be $\sim 50 \%$. Thus approximately 35,000 substrates must be manufactured. Twenty-four ovens will be used for NuSTAR (with additional ovens ready as spares) as compared to the 12 ovens currently used for $H E F T$. With a production capability of over 20 substrates per week per oven based on experience with HEFT, the requirement for NUSTAR can be achieved in less than 18 months. By the time a formal decision to proceed to Phase B of flight development is made for NuSTAR (currently scheduled for early 2006), we will have half the substrate production capacity required for NuSTAR already in place. The necessary procurements for the cutting and metrology stations and for the additional ovens will be let at the beginning of Phase B and the facility buildup will be complete within 60 days.

\subsection{Multilayer coating}

Multilayer coatings are required to enhance hard X-ray $(>10 \mathrm{keV})$ reflectivity with a broad energy acceptance. A multilayer structure is a stack of thin layers of alternating materials designed so that the small reflections from each layer add in phase. Depth-graded multilayers vary the bi-layer thickness so that different layers are optimized to reflect 
different wavelengths, providing broadband response. Depth-graded multilayers are applied to the optical surfaces in a production coating facility at DNSC that uses planar magnetron sputtering. The specific multilayer design depends on graze angle, which scales with the optic radius. The 130 shells are divided into 20 groups optimized for a particular range of graze angles.

Before coating, the substrates are cleaned using a three step ultrasonic process and sorted by radius for application of the appropriate design. The DNSC facility can coat $0.8 \mathrm{~m}^{2}$ in substrate surface area in a single run (pump-down, coating and venting of the chamber); each run is a one-day operation. Producing the required number of substrates consistent with the NuSTAR schedule requires coating $1.9 \mathrm{~m}^{2} /$ week, compared to the $4 \mathrm{~m}^{2} /$ week capability, providing significant margin. An additional facility at Columbia provides backup and coating capability for prototypes and engineering models. Quality is assured for each coating run by including a silicon wafer as a witness that is characterized at $8 \mathrm{keV}$ for reflectance spectra. These reflectance data, as well as housekeeping data from the coating chamber are automatically logged to the optics database, also updated to reflect the coating applied to each substrate. The material combinations for the coatings depend on graze angle - the inner 14 radius groups (about 2/3 of the geometric area) are coated with $\mathrm{Pt} / \mathrm{SiC}$, and the remaining are coated with $\mathrm{W} / \mathrm{SiC}$. Refer to Jensen et. al for a more complete discussion of NuSTAR multilayers.

\subsection{Optics Assembly}

The coated substrates are assembled into an optic at an existing facility, developed for HEFT at Colorado Precision Products Incorporated (CPPI) in Boulder, CO. Our unique error-compensating, monolithic assembly and alignment (EMAAL) procedure involves constraining the mirror segments to precisely machined graphite spacers that run along the optical axis. In this process, the nominally cylindrical glass segments are forced to a conical form, and in the process, radial mismatches and some small twists in the glass are removed. In order to achieve large effective area, concentric layers of glass are stacked on top of each other starting with a central mandrel. Graphite spacers are first epoxied to the mandrel and then precisely machined to the correct radius and angle. Next, a layer of glass and second layer of spacers are epoxied to the first set of spacers. These spacers are then machined to the appropriate radius and angle. This process is repeated until the requisite number of layers is assembled.

A key point of this process is that each layer of spacers is machined with respect to the optic axis and not the last layer of glass. In this way, there is never any stack-up error during the telescope fabrication. Each layer requires one workday to complete. Two machines operating in parallel produce two layers per day. In this way, four NuSTAR optics modules can be assembled in just over one year.

\section{OPTICS DEVELOPMENT}

In addition to building up our infrastructure specifically for NuSTAR, we continue an independent program of optics development for other future missions, which is funded by a separate NASA grant. While no further development is required for NUSTAR, several components to this independent research program are becoming mature enough to have an impact on NuSTAR. Each focuses on improving the yield of high quality substrates with an incremental impact on the overall optic performance. As such, and of these steps could easily be incorporated into the NuSTAR program with no change to the fundamental design. In this section we will detail multiple strategies that we are actively pursuing to further improve the yield of high quality substrates.

\subsection{Raw Glass Selection}

Mirror fabrication begins with microsheet glass originally developed for the flat panel display industry. This type of glass is produced (by both Schott and Corning) using an overflow process in which the glass is vertically drawn under the influence of gravity. Since there is never physical contact with the glass, the resulting product has extraordinarily good flatness and uniformity, particularly in the draw direction. We begin by placing a glass micro-sheet on top of a concave mandrel inside of the oven. The draw direction of the glass is aligned with the mandrel's axis of symmetry since the performance of the optic is dominated by slope errors along the optical axis. Since slope errors in the azimuth direction of the mirror are suppressed by the ratio of the mirror radius to the telescope focal length (i.e., a factor of 100), the poorer intrinsic performance of the glass perpendicular to the draw direction will not affect the overall performance of the mirror. As the oven is heated to the appropriate forming temperature, the glass begins to form into the mandrel under the influence of gravity. Just before the glass touches the mandrel surface, the forming process is

\footnotetext{
${ }^{\ddagger}$ http://www.coloradoprecision.com/
} 
terminated by lowering the oven temperature. In this way, near net shaped substrates are produced without perturbing the initial X-ray properties of the glass micro-sheet. Thus, flat glass with higher quality initial figure will produce better performing substrates.

A database is used to track each piece of glass (over 12,500 to date) from slumping to eventual mounting. From this, we have found that every oven and every mandrel has produced both high and low quality glass. However, we have discovered that some lots of glass provide statistically better or worse glass than others. We have long used a monochromatic green light source to verify the draw direction of the glass. Thickness variations in the glass (which typically only occur perpendicular to the draw direction) appear as fringes under this light. We have systematically compared glass from lots that produced superior and inferior slumped glass, and we have found that the fringe patters of the superior glass had much straighter fringe lines along the draw direction. Using these interferograms we have begun establishing a quantitative metric for acceptable initial flat glass. Moreover, it also appears that there may be a correlation between this draw quality effect and glass roughness (as measured by $8 \mathrm{keV} \mathrm{X-rays).} \mathrm{Surface} \mathrm{roughness} \mathrm{is,}$ of course, crucial for high reflectivity in hard X-ray optics.

We have begun working with Schott to address this issue and meet our requirements. Schott has agreed to work with us to develop a selection procedure for the best glass. As part of this proposal, they will provide glass samples from their daily production. Before slumping the glass, we will characterize the glass under monochromatic light and with X-rays. The raw glass acceptance criteria developed under this effort will likely lead to improved glass yield and significant savings in terms of both time and money.

\subsection{Substrate Selection}

We continue developing techniques to improve our ability to 'cherry-pick' the best substrates and accurately predict their mounted performance. ${ }^{\S}$ The surface topology of each slumped shell is evaluated in its freestanding state using our laser reflectometer. We have experimentally demonstrated that there are no internal stresses in the glass that cause the surface figure of a section cut from the original complete shell to change. Thus, we can virtually 'cut' a slumped glass segment to any size in software and predict its mounted performance. In this way we are able to choose the best performing section of only the highest quality substrates before investing the time and effort necessary to actually cut them.

The power of this selection process to achieve high performance is illustrated in Figure 5. Here, the performance histograms of $200 \mu \mathrm{m}$ AF45 cut to different geometry are compared. A steady improvement in performance is achieved first by choosing a smaller section of glass (the best $45^{\circ}$ octant section of the original $72^{\circ}$ quint shell) and then by choosing only the best virtually mounted octant pieces. The quint distribution ( $\left(1^{\text {st }}\right.$ analysis $)$ is what we would expect if we would mount all 2150 substrates in the geometry used for HEFT, with a total performance of 65". If we select the best octant section of all 2150 substrates ( $2^{\text {nd }}$ analysis), then we would expect to achieve a combined performance of 49". Finally, if we build an optic using only the substrates with $<60$ " performance and discard the rest of the substrates, then we expect to achieve a improved performance of 37", which exceeds the 40" goal of NuSTAR. This represents a 59\% yield. We can achieve even higher performance by imposing a more stringent selection criterion, or we can allow for a higher yield by accepting a slightly lower performance.

Until recently we have relied on 'virtually mounting' freestanding glass with relatively simple software. This procedure has been reliably used to predict the average mounted performance of several prototypes and the HEFT flight optic on an ensemble basis. However, it does not accurately predict the mounted performance on a shell-by-shell basis. While we are still improving this software-based technique for glass selection we are also employing hardware solutions whereby shells are mounted in fixtures that faithfully represent the mount process. These two methods are detailed in the subsections below.

\subsubsection{Virtual Mount Software}

We have developed a relatively simple code that virtually mounts the glass based on the freestanding laser data. It assumes that the glass is mounted perfectly (or to an arbitrary input figure value) directly on top of the spacers and uses a simple interpolation procedure to force the axial figure to return to its freestanding state away from the spacers. Twist in the glass is dampened relative to the separation between spacers (i.e., more spacers results in less phase-error and a lower overall HPD). Using finite element analysis (FEA) software (Pro-E and Ansis) we have begun to investigate more realistic mount simulations. We have started with simple models of constraining a shell of one radius to a slightly

\footnotetext{
${ }^{\S}$ Refer to Koglin et. $\mathrm{al}^{5}$ for a more detailed description of the glass selection procedure.
} 
different radius. We plan to continue this line of investigation by simulating the mounting process on imported surface topology data.

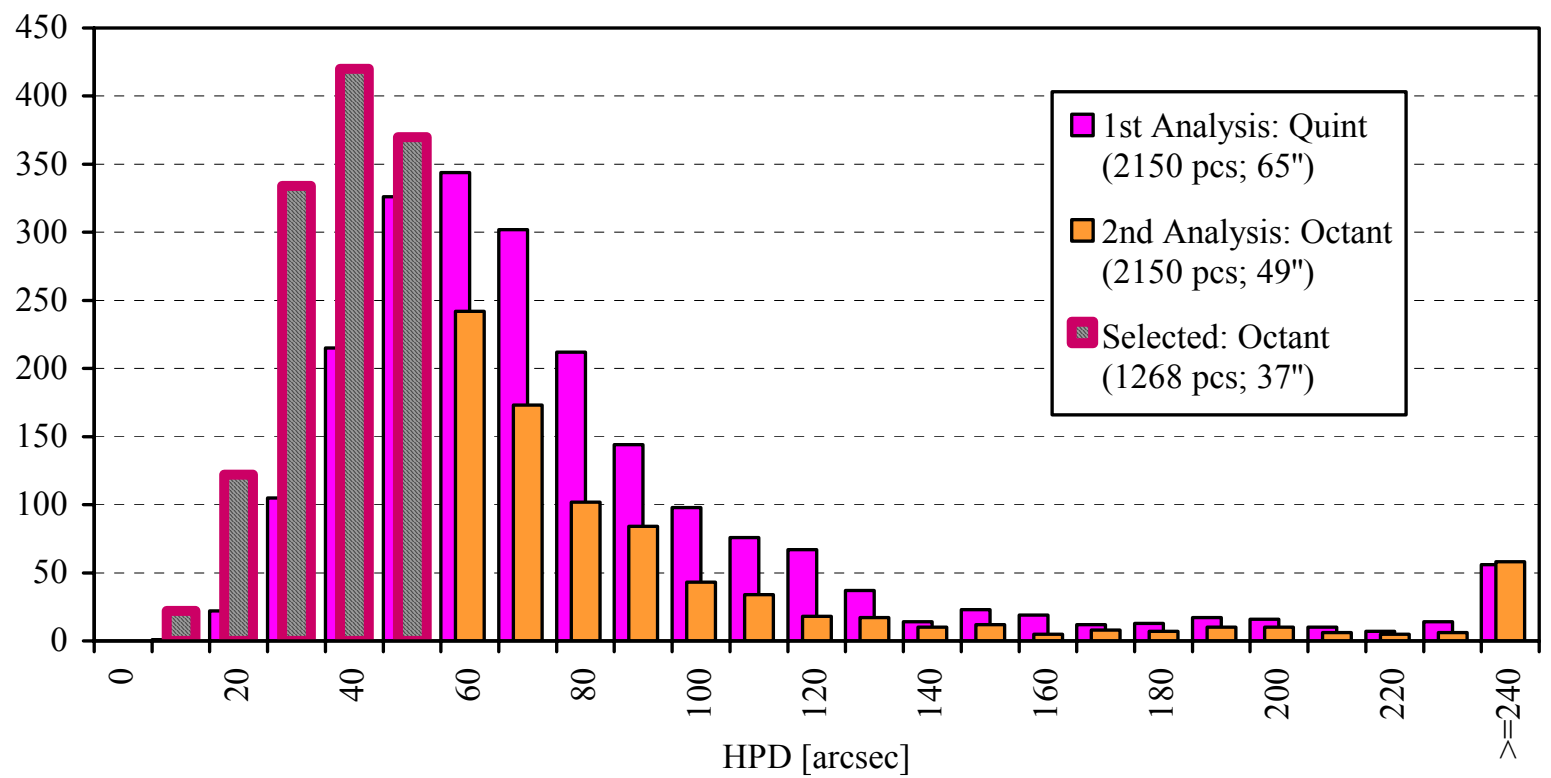

Figure 5: Performance histogram of $200 \mu \mathrm{m}$ glass segments highlighting the two-step substrate selection process. The number of substrates and predicted HPD performance is listed for each step.

\subsubsection{Surrogate Mounting Fixtures}

Parallel to our effort to improve our software selection process using freestanding data, we have begun investigating surrogate mount fixtures to simulate the EMAAL mounting process. Our original surrogate mount fixture, shown in Figure 6, uses a similar mounting to that used in the EMAAL process. Once the shell is mounted in this way, metrology can be performed using either the laser reflectometer (as shown in Figure 6) or a contact probe such as an air-bearing Linear Variable Differential Transformer (LVDT). Selecting glass in this fashion will be a far more reliable means to ensure good mounted performance. Further, nondestructive measurements (i.e., no epoxy is used) can be performed repeatedly on the same substrate using different approaches (e.g., spacer size and configuration, mounting force, etc...).

These initial tests have been encouraging, and we have begun investigating alternative mounting fixture designs. The vacuum mount fixture shown in Figure 7 has the advantage that it is simpler to mount the substrate and the entire front

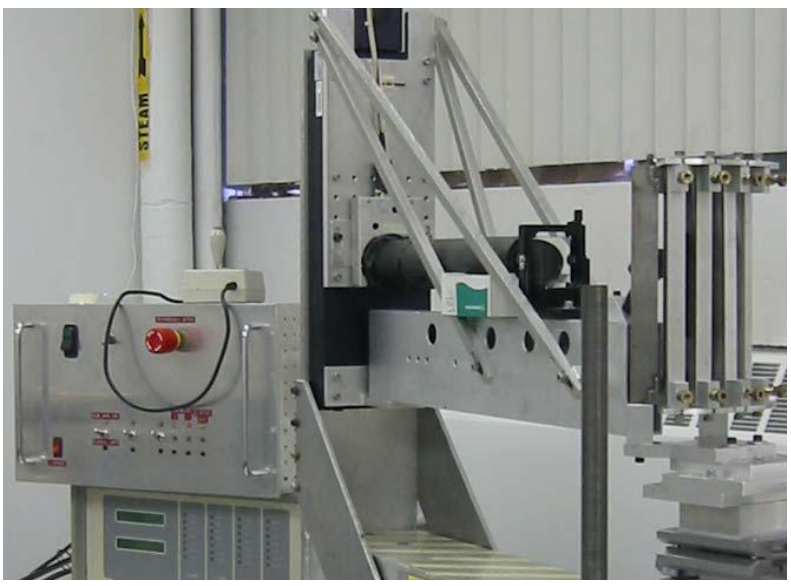

Figure 6: Themally formed glass mounted in a surrogate mount (far right) on the laser reflectometer.

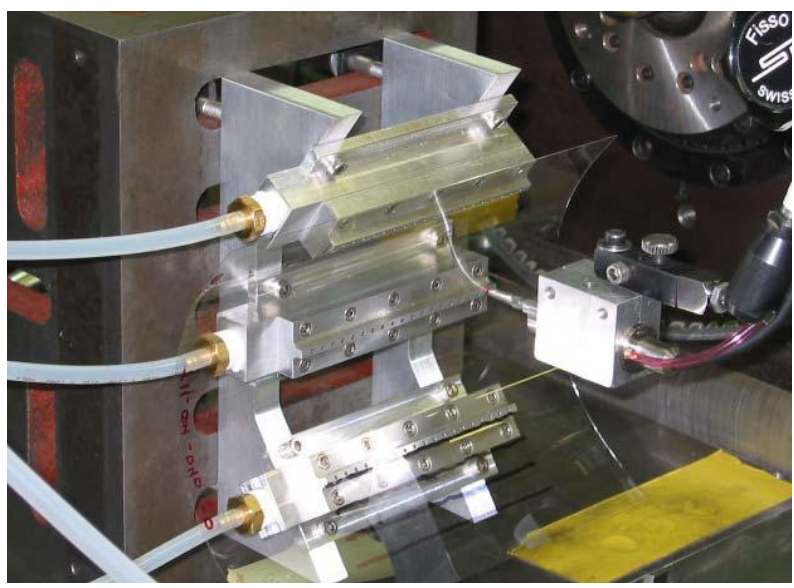

Figure 7: Vacuum mount fixture. 
reflective surface is available including directly on top of the spacers. Initial tests of this fixture using the LVDT are promising, and we have begun to test the accuracy of this approach by comparing surrogate mount results with the results of the same shells EMAAL mounted on prototype optics.

\subsection{Substrate Production Summary}

In this section, we have described several components of our independent optics develop program that are beginning to reach maturity. We are also investigating other slumping techniques and seek to utilize even thinner glass substrates. The NuSTAR optics budget, production schedule and performance requirements are based on current proven technology and do not require any further development. However, as we await the decision to proceed to Phase $\mathrm{B}$ for NuSTAR, any proven process enhancements that improve the yield of high performance substrates without adding additional cost or adversely affecting the production schedule will naturally be incorporated into the NuSTAR production design. In fact, each of the processes discussed in this section are focused on decreasing production time and cost through an improvement in glass yield.

\section{ENVIRONMENTAL TESTING}

We continue to address issues related to space qualification through simulation and prototype testing to ensure there will be no significant issues encountered during the full qualification testing scheduled to be performed during Phase B. This includes stability tests under both thermal and acoustic/vibration loads.

\subsection{Acoustic Test}

The structural integrity of a NuSTAR prototype was demonstrated through acoustic testing in July 2004. This two-layer prototype was built using $200 \mu \mathrm{m}$ thick substrates with a nominal radius of $110 \mathrm{~mm}$ (slightly greater than the central NuSTAR radius). Each of the 64 mirrors was coated with the appropriate $\mathrm{W} / \mathrm{Si}$ multilayer coating. The coated mirrors were mounted in an octant design configuration on a Titanium mandrel. Analysis of the LVDT data, which was recorded for both layers as the optic was being assembled, yields a performance of 50" HPD. After assembly, the acoustic test prototype was shipped to DNSC for X-ray calibration. High-resolution X-ray measurements at $8 \mathrm{keV}$ were performed every degree (refer to Koglin et. al for a general description of the X-ray calibration procedure).

The acoustic test prototype then shipped to Jet Propulsion Laboratory, where it was freely suspended in a 10,900 cu. ft. acoustic chamber (c.f., Figure 9) using bungee cords. Four control microphones were located spatially in proximity to the optics module. The optic was first subjected to the Pegasus Launch levels plotted in Figure 8 for 60 seconds. No physical damage to the prototype was observed to have occurred during this test. The optic was then similarly subjected to the higher developmental levels (also plotted in Figure 8). No physical damage was observed during this more stringent test. The acoustic test prototype was again returned to DNSC and the same X-ray calibration procedure was

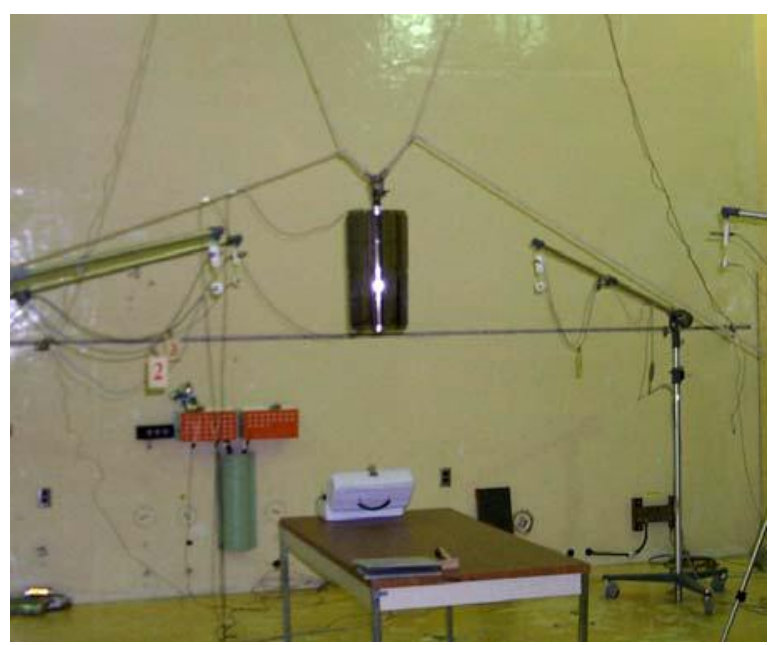

Figure 9: $N u S T A R$ acoustic prototype (center of picture) inside acoustic chamber with microphones.

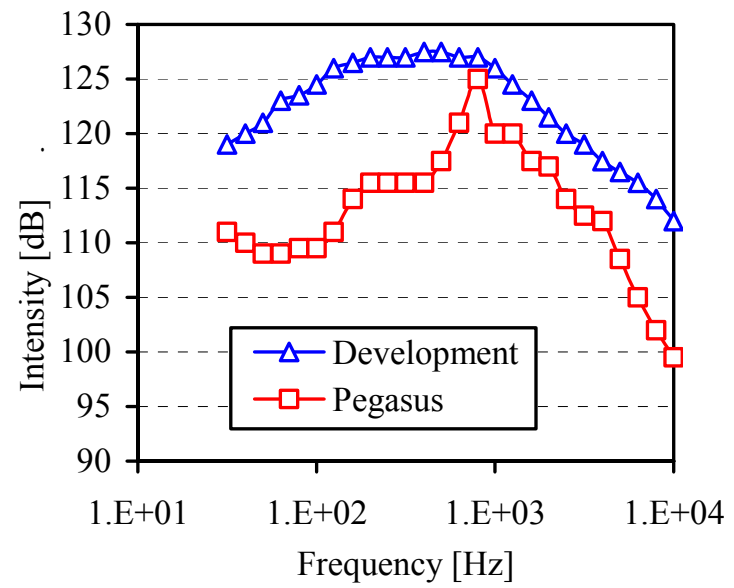

Figure 8: Acoustic test specifications for development and Pegasus requirements. 
repeated. X-ray metrology data indicates that no change occurred in the performance of this optic during the acoustic test. The fraction of $\mathrm{X}$-rays enclosed within a circle at the focal plane is plotted versus the diameter of that circle in Figure 10. The half power diameter (HPD) is the diameter in which $50 \%$ of the Xrays (i.e., power) are enclosed. The combined HPD was found to be $49 "$ and $50 "$ before and after the acoustic test, respectively - well within the estimated systematic uncertainty of $5 "$ in the X-ray measurements.

\subsection{Thermal Test}

We have built and begun using the thermal test chamber shown in Figure 11, which is located in the same building at CPPI where the telescopes are assembled. The system is designed to maintain one-degree temperature stability, employing multiple temperature sensors and a dedicated DAQ system. In an initial trial of our thermal test chamber, a small prototype was built using $200 \mu \mathrm{m}$ thick glass with a nominal radius of $80 \mathrm{~mm}$. Eight uncoated substrates were mounted in an octant configuration on an Aluminum mandrel. LVDT measurements were performed every $2.5^{\circ}$ on each of these mounted shells. Then, a series of thermal bake cycles were executed at $+5^{\circ},+10^{\circ}$, and $+15^{\circ} \mathrm{C}$ above the ambient temperature $\left(20^{\circ} \mathrm{C}\right)$ in which the optic was build. For each cycle, six thermal probes were attached to positions on both the shells and the mandrel. The optic was then wrapped in aluminum foil and placed in the thermal chamber. The temperature in the chamber was slowly elevated to the cycle temperature and held for one hour. The optic was then cooled to room temperature, unwrapped and remounted on the assembly machine so that LVDT metrology could be performed. The LVDT data was acquired each cycle using the same procedure as the original measurement. This procedure was repeated for each of the three temperature cycles. The optic was then subjected to a $0^{\circ} \mathrm{C}$ cold cycle $\left(-20^{\circ}\right.$ below ambient temperature) .
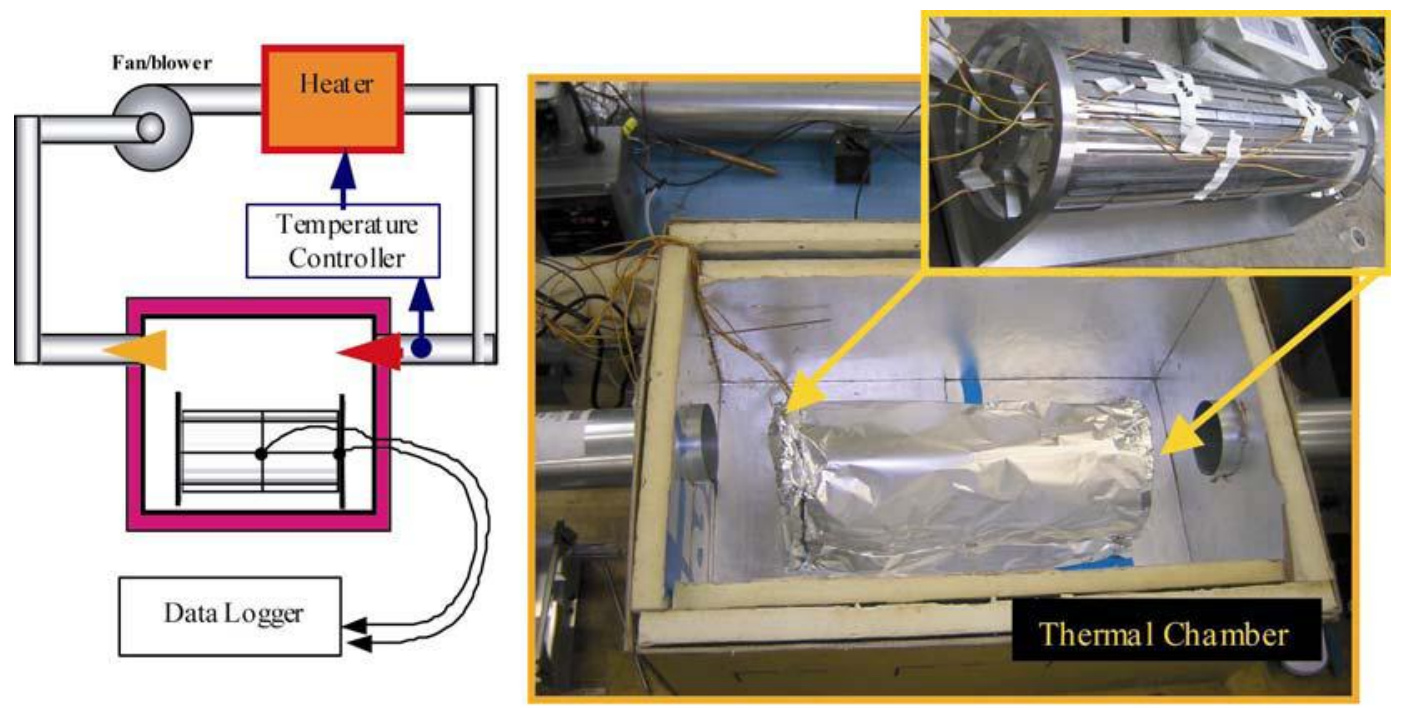

Figure 11: Thermal test chamber design. The general system is illustrated to the left. The pictures to the right show temperature probes attached to a prototype optic, which was subsequently wrapped in aluminum foil and placed in the thermal test chamber.

There was little change in the surface height metrology as is illustrated in Figure 12, a plot of axial LVDT measurements performed along the same azimuth position after each thermal cycle and compared with the original measurement. The total performance of the optic remained consistent throughout each cycle with an average HPD performance of $59 \pm 5$ " for 
the five measurements, within the uncertainty (5") of the LVDT measurement system and consistent with the 62" predicted performance based on analysis of the laser scanner data recorded for the unmounted substrates. We will continue performing more extensive thermal test cycles on future prototypes with more mirrors and at different radii until we move into Phase B, at which time full qualification testing will be conducted.

\section{SUMMARY}

As the first satellite mission to employ focusing optics in the hard X-ray band, NUSTAR is poised to usher in a new period of discovery. In this paper, we have presented an overview of the NuSTAR optics design and production process and outlined the steps we are taking in preparation for flight development. The NuSTAR production design is based on demonstrated technology and does not require further development. However, any proven process enhancements that improve the yield of high performance substrates without adding additional cost or adversely affecting the production schedule will be incorporated into the NUSTAR design. We have presented an overview of several components of our independent optics development program that may impact NuSTAR in this way. Finally, we have presented environmental test results that are being conducted in preparation for full space qualification of the NuSTAR optics.

\section{ACKNOWLEDGEMENTS}

This work is supported by a NASA grant to Columbia University: NAG5-5260 (Thermally Formed Optics for X-Ray and Gamma-Ray Astronomy), and by NASA grants to the California Institute of Technology with sub-awards to Columbia: Caltech No. 1019776 (High Energy Focusing Telescope) and Caltech No. 1046806 (Critical Technologies for the Constellation Hard X-Ray Telescope).

\section{REFERENCES}

${ }^{1}$ C. M. H. Chen, W. R. Cook, F. A. Harrison, J. Y. Lin, P. H. Mao and S. M. Schindler, "Characterization of the HEFT CdZnTe pixel detectors", Proc. SPIE, 5198 (2004) 9-18.

${ }^{2}$ J.E. Koglin, F.E. Christensen, J. Chonko, W.W. Craig, T.A. Decker, C.J. Hailey, F.A. Harrison, C.P. Jensen, M. Sileo, D.L. Windt and H. Yu, " Development and Production of Hard X-ray Multilayer Optics for HEFT," Proc. SPIE 4851 (2003) 607-618.

${ }^{3}$ J.E. Koglin, H.C. Chen, F.E. Christensen, J. Chonko, W.W. Craig, T.R. Decker, M.A.Jimenez-Garate, C.J. Hailey, F.A. Harrison, C.P. Jensen, M. Sileo, D.L. Windt and H. Yu, "Development of Precision Hard X-ray Multilayer Optics with Sub-Arcminute Performance," Proc. SPIE 4851 (2003) 673-683.

${ }^{4}$ J.E. Koglin, C.M.H. Chen, F.E. Christensen, J. Chonko, W.W. Craig, T.R. Decker, K. S. Gunderson, C.J. Hailey, F.A. Harrison, C.P. Jensen, K.M. Madsen, M. Stern, D.L. Windt and H. Yu, E. Ziegler, "Production and calibration of the first HEFT hard X-ray optics module", Proc. SPIE 5168 (2004) 100-111.

${ }^{5}$ J.E. Koglin, C.M.H. Chen, J.C. Chonko, F.E. Christensen, W.W. Craig, T.R. Decker, C.J. Hailey, F.A. Harrison, C.P. Jensen, K.K. Madsen, M.J. Pivovaroff, M. Stern, D.L. Windt, E. Ziegler, "Hard X-ray Optics: From HEFT to NuSTAR”, Proc. SPIE 5488 (2004) 856.

${ }^{6}$ C. P. Jensen, F. E. Christensen, A. Jensen, K. K. Madsen, "X-ray study of $\mathrm{W} / \mathrm{SiC}$ and $\mathrm{Pt} / \mathrm{SiC}$ multilayers for the NuSTAR hard-x-ray telescope", Proc. SPIE 5900-07 (2005). 